\title{
GEOMETRICAL CLASS AND DEGREE FOR SURFACES IN THREE-SPACE
}

\author{
THOMAS BANCHOFF \& NICOLAAS H. KUIPER
}

Dedicated to Professor Buchin Su on his 80 th birthday

\section{Definitions and theorems}

We consider immersions and embeddings $f: M \rightarrow E^{3}$ of a closed surface $M$ into euclidean three-space $E^{3}$, in the smooth $\left(C^{\infty}\right)$ and the polyhedral (= piecewise linear $=P L$ ) category. Occasionally we will mention the topological category.

For any line $l$ in $E^{3}$ we can choose the parallel projection $\pi_{l}$ of $E^{3}$ into an orthogonal plane $\alpha_{l}$. We will use polar coordinates $(r, \theta)$ and euclidean coordinates $(u, v)=(r \cos \theta, r \sin \theta)$ around $(0,0)=l \cap \alpha_{l}$ in that plane.

The line $l$ is said to be transversal to $f$ in case there is a neighborhood $U_{p} \subset M$ for any point $p \in f^{-1}(l)=\{q \in M: f(q) \in l\}$, for which the projection

$$
\pi_{l} \circ f: U_{p} \rightarrow \alpha_{l}
$$

is an isomorphism (diffeomorphism, etc.) onto its image, which image we can assume to be a round disc $r<\delta$ for some $\delta>0$. Then we define the geometrical degree $d=d(f)$ of the surface $f$,

$$
d(f)=\sup _{l \text { transversal }} d_{l}(f) \leqslant \infty,
$$

to be the least upper bound of the number of points $d_{l}(f)$ of $f^{-1}(l)$ for all transversal lines $l$. The number $d_{l}(f)$ is constant on each component of the open dense subspace of $f$-transversal lines in the Grassmann manifold $\mathrm{Gr}$ of all lines in $E^{3}$. Any transversal line can be moved into the position of any other one in such a way that the number of points $d_{l}(f)$ changes by an even number at isolated times at which the line is not transversal. In $E^{3}$ we can moreover

Received December 17, 1981. The authors had the benefit of conversations with E. Calabi, Y. Kergosien, W. Kühnel, W. Pohl and R. Thom. 
move $l$ away from $f(M)$, so that eventually $d_{l}(f)$ is zero. We easily conclude

Lemma 1. The geometrical degree $d(f) \leqslant \infty$ is even for smooth or $P L$ immersed closed surfaces $f: M \rightarrow E^{3}$. If $d(f)=2$, then $f$ is an embedding of the 2-sphere onto a convex surface. The degree $d(f)$ is finite for a PL-surface.

Observe also that if $h(x, y, z)$ is a polynomial of degree $m$ in euclidean coordinates $x, y, z$ for $E^{3}$, and $f: M \rightarrow E^{3}$ is an embedding into the real algebraic variety with equation $h(x, y, z)=0$, then

$$
\text { geometrical degree } \dot{d}(f) \leqslant \text { algebraic degree } m \text {, }
$$

(over $\mathbf{C}$ the two degrees are equal).

In order to define the geometrical class of a surface $f$, we examine (and define) the "number of tangent planes $c_{l}(f)$ " through a general transversal line $l$ as follows. The function $\tilde{\theta}_{l}=\tilde{\theta}=\theta \circ f$ modulo $\pi$, in terms of the polar coordinate $\theta$, is well defined on $M \backslash f^{-1}(l)=\{q \in M: f(q) \notin l\}$ with values in the circle $\mathbf{R} / \pi \mathbf{Z}$. It has no critical points near $f^{-1}(l)$ and it is constant on each plane through $l$. We call the transversal line $l$ general for a smooth surface in case the function $\tilde{\theta}_{l}$ is nondegenerate. We denote by $c_{l}^{+}(f)$ the number of critical points with relative extreme value (maximum or minimum), and by $c_{l}^{-}(f)$ the number of other critical values (saddle points), and we put $c_{l}(f)=$ $c_{l}^{+}(f)+c_{l}^{-}(f)$.

We call the transversal line $l$ general for a piecewise linear surface (the pieces are the simplices of some triangulation of $M$ ) in case the function $\tilde{\theta}_{l}$ has only isolated critical points, say $c_{l}^{+}(f)$ with relative extreme values, $c_{l}^{-}(f)$ others, with $c_{l}(f)=c_{l}^{+}(f)+c_{l}^{-}(f)$ as "total number of tangent planes". But in the $P L$-case the level curve $\left\{q \in M: \tilde{\theta}_{l}(f(q))=\gamma\right\}$ of a critical nonextreme value $\gamma$ is seen to consist near the isolated critical point $p$ of an even number $2(m+1)$ of straight line segments $(m \geqslant 1)$ each ending at $p \in M$, and then that critical point must be counted with multiplicity $m$ in $c_{l}^{-}(f)$ in order to agree with the Morse theory to be applied later. Compare Kuiper [6].

In the $C^{\infty}$ and $P L$-categories the general lines form an open dense set in $\mathrm{Gr}$, and $c_{l}^{+}(f), c_{l}^{-}(f), d_{l}(f)$ are constant on each component of this subspace.

Definition. The geometrical class of the immersion $f: M \rightarrow E$ is the least upper bound

$$
c=c(f)=\sup _{l \text { general }} c_{l}(f)
$$

We also put

$$
\begin{aligned}
& c^{+}(f)=\sup _{l_{\text {general }}} c_{l}^{+}(f), \\
& c^{-}(f)=\sup _{l_{\text {general }}} c_{l}^{-}(f) .
\end{aligned}
$$


Clearly

$$
c^{+}(f)+c^{-}(f) \geqslant c(f) \geqslant c^{ \pm}(f) .
$$

In $\$ 2$ we prove the main theorem relating class and degree:

Theorem 1. For any smooth or polyhedral immersion $f: M \rightarrow E^{3}$ of a closed surface, we have

$$
c(f) \geqslant d(f)-\chi(M)
$$

where $\chi(M)$ is the Euler characteristic of $M$.

Our interest in the class of a surface originated in its relation to tightness. For a map of a closed surface into euclidean space tightness has a simple characterization. The map $f: M \rightarrow E^{3}$ is tight if the set

$$
f^{-1}(h)=\{q \in M: f(q) \in h\}
$$

is connected for any closed half space $h$ bounded by a plane. A smooth immersion $f$ in $E^{3}$ can be shown to be tight if and only if the total absolute curvature

$$
\frac{1}{2 \pi} \int|K d \sigma|
$$

which is $\geqslant 4-\chi(M)$, attains its minimal value $4-\chi(M)$. See [6]. We also prove, in $\S 2$,

Theorem 2. The geometrical class of a TIGHT smooth or polyhedral immersion $f: M \rightarrow E^{3}$ of degree $d(f) \geqslant 4$ satisfies the equality

$$
c(f)=c^{-}(f)=d(f)-\chi(M) .
$$

By Lemma 1 the assumption $d(f) \geqslant 4$ only excludes convex surfaces $\left(S^{2}\right)$. If $l$ is a general line which does not meet the convex hull $\mathcal{H} f(M)$ of $f(M)$, then tightness of $f$ is seen to imply $c_{l}^{+}(f)=2$, as there could not be more than two "extreme" tangent planes through $l$. Then by the Morse equality $c_{l}^{+}(f)-$ $c_{l}^{-}(f)=\chi(M)$, we obtain

$$
c_{l}(f)=4-\chi(M) .
$$

Even so $c(f)$ can take bigger values as seen in the end of $\S 4$.

Theorem 2a. For a tight smooth immersed surface $f: M \rightarrow E^{3}$, if the Euler characteristic $\chi(M) \neq 2$, then the geometrical class can be made as large as we please by a $C^{2}$-small perturbation, while preserving tightness.

This answers problem 4 in [7] in the negative.

In the rest of the paper we are interested in immersions and embeddings $f$ for which $d(f)$ and $c(f)$ are small, and which are tight as well if possible. In the polyhedral case and for orientable surfaces all wishes can be attained. 
Theorem 3. There are tight polyhedral imbeddings $f: M \rightarrow E^{3}$ of geometrical degree 4 and class $4-\chi(M)$ for any orientable surface $M$ of genus $g \geqslant 0$.

Construction. For $g=0$ we take the surface of a cube. For $g=1$ we delete from the cube the convex hull of the union of two concentric equally large squares in opposite faces, and take the boundary of the remaining ring. See Fig. 1a.

For $g \geqslant 2$ we proceed as follows. See Fig. 1 b. Let $B_{1}$ be the convex body which is a vertical cylinder with basis the two-disc of a regular $g+1-g \circ n$, $g \geqslant 2$, and constant height. Consider in each vertical rectangular face a concentric rectangle $D_{i}, i=0,1, \cdots, g$, and let these rectangles all have the same size. The convex hull

$$
B_{2}=\mathcal{H}\left(D_{0} \cup D_{1} \cup \cdots \cup D_{g}\right)
$$

is a second convex body. Then the boundary of the closure of $B_{1} \backslash B_{2}$, $f(M)=M=\partial \overline{\left(B_{1} \backslash B_{2}\right)}$, is a polyhedral, tight(!) surface of genus $g$. Its geometrical degree is $d(f)=4$, because $M$ is contained in $\partial B_{1} \cup \partial B_{2}$, the union of two convex surfaces, and its class is $4-\chi(M)$ by Theorem 2 .

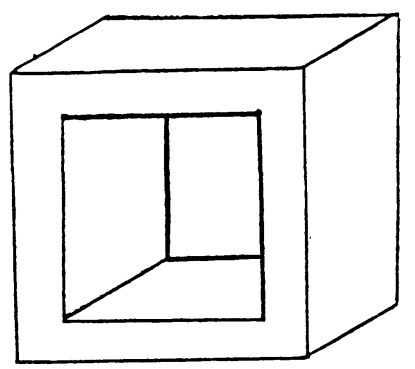

FIG. 1a

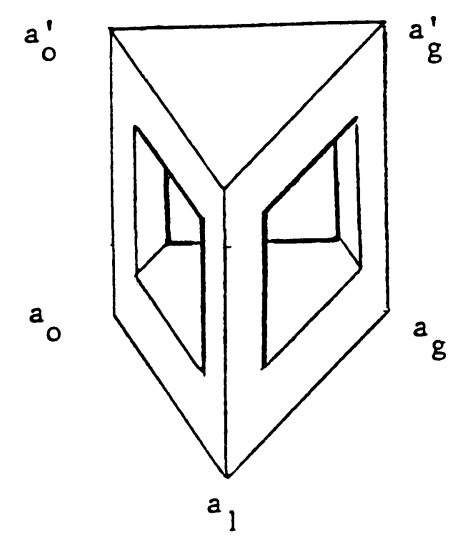

FIG. 1b

Nonorientable closed surfaces $M$ seem to have no immersions of gemetrical degree 4 , and we can prove that for $\chi(M)$ odd. However if we allow "locally stable" maps (generalizing immersions), we do have examples for the projective plane and the Klein bottle. Without going into the definition we recall that a smooth or $P L$ map $f ; \mathbf{R}^{2} \rightarrow \mathbf{R}^{3}$ is stable but not an immersion at $0 \in \mathbf{R}^{2}$, if it is homeomorphic near $0 \in \mathbf{R}^{2}$ to a cone on a circle immersion with one transversal self intersection in a plane $\alpha$ not containing $f(0)$. 


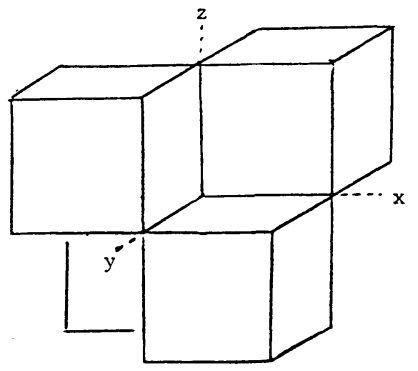

FIG. 2a

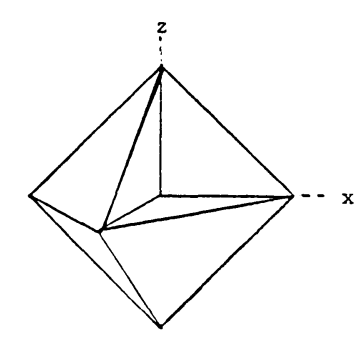

FIG. 2b

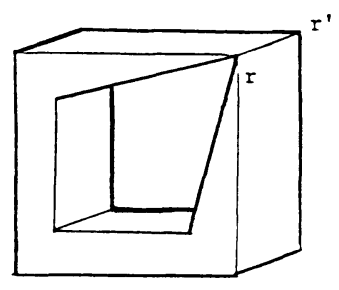

FIG. 2c

In Fig. 2a we have the Petrobras surface a $P L$-stable map of the real projective plane onto the boundary of the union of four cubes with side 1, placed as indicated in the cube with equation $\sup \{|x|,|y|,|z|\} \leqslant 1$. Stable singularities are at $(x, y, z)=( \pm 1,0,0)$, etc. There is a triple point of the surface at $(0,0,0)$ where the coordinate plane parts of the surface meet. Self-intersections are on the coordinate axes. The geometrical degree is clearly 4 , but the map is not tight.

In Fig. $2 b$ we restrict the body of Fig. $2 a$ to the part

$$
\sup _{e^{2}=f^{2}=1}|x+e y+f z| \leqslant 1 .
$$

The surface (boundary) then obtained determines again a locally stable polyhedral map of a real projective plane, of geometrical degree 4, which is seen to be tight as well. Also $c(f)=3$. This "heptahedron" is found already in [11, Fig. 288].

In Fig. 2c we suggest a tight locally stable polyhedral map of the Klein bottle with geometrical degree 4 and class 4 . The self intersection segment $r r^{\prime}$ has stable singularities at its end points $r$ and $r^{\prime}$. (Observe that the set in Fig. $2 \mathrm{c}$ is not the image of a locally stable map of a torus.)

In the rest of the paper we give examples for smooth surfaces.

Theorem 4 (§3). There are smooth (even locally algebraic) embeddings in $E^{3}$ of geometrical degree 4 for orientable closed surfaces of any genus $g \geqslant 1$.

This was announced in [10] by $R$. Thom as an insight of E. Calabi.

We recall also in $\$ 3$ that there is a real algebraic model (surface) of algebraic degree 4 for any orientable surface of genus $g \leqslant 7$.

Theorem 5 (§4). There are smooth (even locally algebraic) TIGHT embeddings in $E^{3}$ of geometrical degree 6 for orientable surfaces of any genus $g \geqslant 2$.

Theorem 6 (\$5). There is a smooth TIGHT embedding in $E^{3}$ of geometrical degree 4 for the orientable surface of genus $g=2$. The given example is a component of an algebraic variety of algebraic degree 5 . 
Conjecture. There is no tight smooth surface of geometrical degree 4 in $E^{3}$ of genus $g>3$. Calabi constructs an example of genus 3 .

We will make some comments about the robustness of the degree and of tightness under $C^{4}$-small perturbations of our examples in $\S 3,4,5$.

Generalizations. 1. Lemma 1 and Theorems 1 and 2 remain true for smooth and $P L$ immersions of a closed surface $M$ into euclidean $N$-space $E^{N}$, $N \geqslant 3$, with suitable modifications in the definitions and proofs. In particular instead of lines $l$ one takes affine subspaces of codimension 2 in $E^{N}$.

2. Theorem 1 remains true for immersions of a closed surface $M$ into real projective $N$-space $\mathbf{R} P^{N}, N \geqslant 3$, with suitable modifications in the definitions. The degree $d(f)$ can be odd, for example $d(f)=1$ for the straight projective plane $\mathbf{R} P^{2}$ in $\mathbf{R} P^{N}$. Surfaces of geometrical degree 2 in $\mathbf{R} P^{3}$ are either convex surfaces of algebraic quadratic hyperboloid surfaces. The last are homeomorphic to the torus. We intend to discuss immersions of nonorientable surfaces of small degree in another paper.

\section{The relation between class and degree}

We recall the Morse equality: If $\varphi: M \rightarrow \mathbf{R}$ is a nondegenerate smooth function on a closed manifold with $\mu_{j}$ critical points of index $j$, then

$$
\sum_{j}(-1)^{j} \mu_{j}=\chi(M) \text {. }
$$

For a surface put $\mu_{0}+\mu_{2}=c^{+}$and $\mu_{1}=c^{-}$. Then

$$
c^{+}-c^{-}=\chi(M)
$$

Suitably modified the same holds for continuous functions with isolated critical points on a closed surface, for example on a polyhedral surface. We now prove

The litte Morse equality: (2.1) holds also for maps of a closed surface $M$ into the circle $S^{1}$ with all critical points isolated (see [12]).

Proof. Let the map $\varphi: M \rightarrow S^{1}\left(=\mathbf{R} / \pi \mathbf{Z}\right.$ say) have $c(\varphi)=c^{+}(\varphi)+c^{-}(\varphi)$ isolated critical points, all other points assumed regular for $\varphi$. Let $[s-2 \varepsilon$, $s+2 \varepsilon], \varepsilon>0$, be an interval of regular values. $\varphi^{-1}(s)$ is a union of $k(\geqslant 0)$ circles, $\varphi^{-1}([s-2, \varepsilon, s+2 \varepsilon])$ is a union of $k$ orientable bands in $M$. Delete these bands and close each of the $2 k$ bounding circles of the rest of $M$ by a cone, so that a new abstract surface $M^{\prime}$ is obtained. Extend the function $\varphi$ over the cones to obtain $\varphi^{\prime}$ so that the only new critical points of $\varphi^{\prime}$ are $k$ relative maxima with value $s-\varepsilon$ and $k$ relative minima with value $s+\varepsilon$, in the 
obvious way. The new function $\varphi^{\prime}$ on the new manifold $M^{\prime}$, for which $\chi\left(M^{\prime}\right)=\chi(M)+2 k$, has invariants:

$$
c^{+}\left(\varphi^{\prime}\right)=c^{+}(\varphi)+2 k, \quad c^{-}\left(\varphi^{\prime}\right)=c^{-}(\varphi) .
$$

As $\varphi^{\prime}$ maps into an interval (embeddable in $\mathbf{R}$ ) the usual Morse equality holds (see [6]):

$$
\chi(M)+2 k=\chi\left(M^{\prime}\right)=c^{+}\left(\varphi^{\prime}\right)-c^{-}\left(\varphi^{\prime}\right)=c^{+}(\varphi)+2 k-c^{-}(\varphi) .
$$

Hence the little Morse equality follows.

Next let $f: M \rightarrow E^{3}$ be a smooth or polyhedral immersion of a closed surface $M$ and $l$ a general line. We prove with $d_{l}=d_{l}(f), c_{l}^{-}=c_{l}^{-}(f)$, etc.:

Lemma 2.1. $c_{l}^{-}=d_{l}-\chi(M)+c_{l}^{+} \geqslant d_{l}-\chi(M)$.

Proof. See $\S 1$ for the meaning of round neighborhoods $U_{p}, p \in f^{-1}(l)$, and of $\tilde{\theta}_{l}=\theta \circ f$. Take out such round neighborhoods $U_{1}, \cdots, U_{d_{l}}, d_{l}=2 m$ from $M$, one for each point $p \in f^{-1}(l)$, and connect $\partial U_{2 j-1}$ and $\partial U_{2 j}$ by a cylinder to obtain a new abstract surface $M^{\prime}$ with (clearly)

$$
\chi\left(M^{\prime}\right)=\chi(M)-d_{l} .
$$

Assume the discs $f\left(U_{2 j-1}\right)$ and $f\left(U_{2 j}\right)$ disjoint, and extend $f$ to get $f^{\prime}$, mapping the cylinders onto tubes around $l$. Then $f^{\prime}\left(M^{\prime}\right)$ avoids $l$, and $\tilde{\theta}_{l}^{\prime}=\theta \circ f^{\prime}$ maps $M^{\prime}$ into the circle $\mathbf{R} / \pi \mathbf{Z}$. We may assume that the critical points of $\theta$ on $f^{\prime}\left(M^{\prime}\right)$ are the same as those of $\theta$ on $f(M)$. Since $\theta_{l}^{\prime}$ is defined at all points of $M^{\prime}$, the little Morse equality holds and we have

$$
c_{l}^{+}-c_{l}^{-}=\chi\left(M^{\prime}\right)=\chi(M)-d_{l} .
$$

Hence Lemma 2.1 follows.

Another proof. The above proof does not apply in case $d_{l}$ is odd, which may happen with immersions into $\mathbf{R} P^{3}$. Therefore we present a second proof. Replace $M$ by a new abstract surface $M^{\prime}$, by taking out the open round disc $U_{p}$ for $p \in f^{-1}(l)$ again, and identifying diametrical points on $\partial U_{p}$. Two such diametrical points have the same value of $\bar{\theta}_{l} \circ f$ in $\mathbf{R} / \pi \mathbf{Z}$. The new manifold $M^{\prime}$ (obtained by $d_{l}$ such "blowing ups", as they are called) has $\chi\left(M^{\prime}\right)=\chi(M)$ $-d_{l}$, and the function $\tilde{\theta}_{l} \circ f$ has the same critical points as on $M($ !); Lemma 2.1 follows as before.

Theorem 1 follows from Lemma 2.1 by taking least upper bounds for general lines $l$ :

$$
c(f) \geqslant c^{-}(f) \geqslant d(f)-\chi(M) .
$$

Proof of Theorem 2. We now assume that $f$ is tight and $d(f) \geqslant 4$. For a general line $l$ which does not meet the convex hull $\mathcal{H} f(M)$ we observed already

$$
c_{l}^{+}=2, c_{l}=4-\chi(M) \text {. }
$$


For a general line $l$ which does meet $\mathscr{H} f(M)$, clearly by definition of tightness, $c_{l}^{+}=0$ and, by Lemma 2.1

$$
c_{l}=c_{l}^{-}=d_{l}-\chi(M) .
$$

Taking upper bounds for all general $l$ we get the equality of Theorem 2:

$$
c(f)=c^{-}(f)=d(f)-\chi(M) .
$$

\section{Smooth (even locally algebraic) orientable surfaces of geometrical degree 4}

A smooth two-sphere or revolution of degree 4. Any tangent line $l$ in an asymptotic direction of a piece of smooth surface of negative Gauss curvature has in general local contact of order three, and a small perturbation of $l$ can give a contribution three to the degree $d_{l}$. Sweeping around such asymptotic tangents it seems hard to obtain $d(f)=4$ for surfaces of high genus $g \geqslant 2$.

It is also interesting to observe what can happen if one rotates a convex curve $\gamma$ in a vertical plane around a disjoint vertical line in that plane. If $\gamma$ is a round circle we get the standard torus of algebraic and geometrical degree 4 . If $\gamma$ is a square with two vertical sides, or a smooth approximation like the curve with equation

$$
|x|^{p}+|y|^{p}=1, \quad p \geqslant 4
$$

we get again an embedded torus with geometrical degree 4, as W. Kühnel observed for $p \geqslant 4$. But if we place the diagonal of the square, or a smooth approximation, parallel to the vertical axis, then the embedded torus of revolution so obtained has geometrical degree at least six. Look and see!

Even so, following a conviction of E. Calabi, we now proceed to get smooth surfaces of degree four of any genus $g$. We start from the following real algebraic variety $M_{0}$ of algebraic degree four in euclidean coordinates $x, y, z$ for $E^{3}$ :

$$
\begin{aligned}
& \left(r^{2}-1\right)^{2}+\varepsilon^{4}(1+\varepsilon)\left(\frac{z+1}{2}\right)^{4}=\varepsilon^{4}, \\
& r^{2}=x^{2}+y^{2}+z^{2}, \quad \varepsilon>0 \text { small. }
\end{aligned}
$$

The planes $z=$ constant are said to be horizontal. $M_{0}$ is contained in the thick two-sphere

$$
S:\left(r^{2}-1\right)^{2} \leqslant \varepsilon^{4}
$$


and also in the convex domain

$$
D:\left(\frac{z+1}{2}\right)^{4} \leqslant \frac{1}{1+\varepsilon}
$$

which for small $\varepsilon>0$ cuts away a cap from the top of the thick two-sphere.

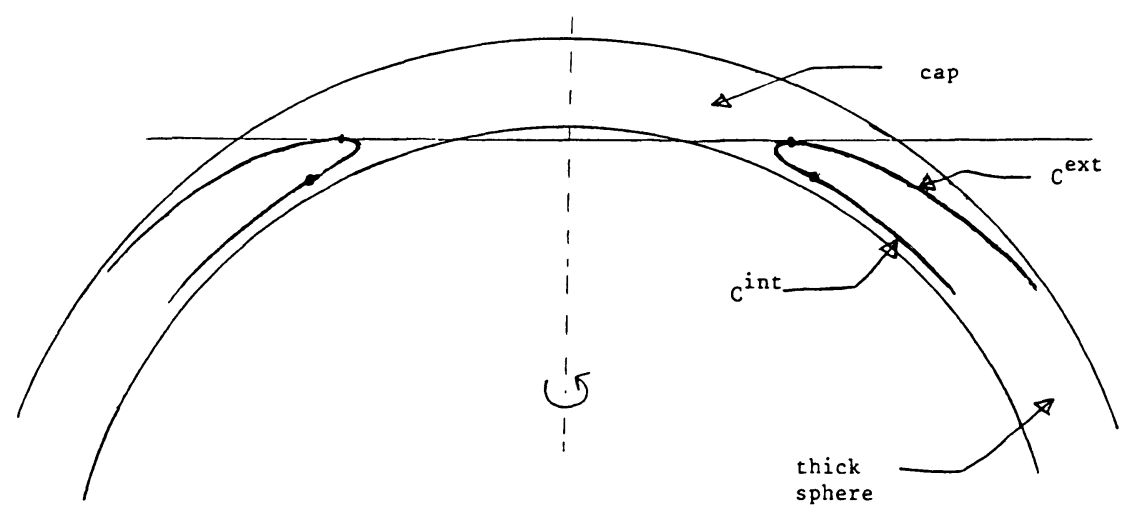

FIG. 3a

Half rays from the origin $(0,0,0)$ give two or no intersection points with $M_{0}$. We have an embedded two-sphere, and a surface of revolution of algebraic and hence geometric degree 4. See Fig. 3a.

The surface has two large convex parts where $K \geqslant 0$; one exterior $C^{\text {ext }}$ and one interior $C^{\text {int }}$. The asymptotic curves in the complement are tangent to the boundary of $C^{\text {ext }}$ where the Gauss curvature is $K=0$. They show a pattern as on the standard torus. It is not generic from the differential point of view as Thom and Kergosien observed in [3].

Near the boundary of $C^{\text {int }}$ the asymptotic curves end in cusps, orthogonal to that boundary. Now for small $\varepsilon$, all asymptotic tangents are nearly horizontal (!). That is why they cannot harm in the surface with respect to degree at points of $M_{0}$ which are far away. We use this in our constructions later. The degree is not influenced if we perturb only $C^{\text {ext }}$ and $C^{\text {int }}$ keeping them convex, and unchanged near their boundaries. We may do this in such a way that for some small $\delta>0$ and $z \leqslant 1-\delta$, the surface coincides there with parts of two concentric spheres with radii $r_{1}$ and $r_{2}$ close to 1 and $\left|r_{2}-r_{1}\right|$ very small.

We call the part outside these two spherical parts a Calabi handle attached to the pair of spheres. For small $\varepsilon>0$, it is as small as we please. The surface is clearly not tight, but is still an embedded 2-sphere of revolution of geometrical degree 4. 
Finally for small $\varepsilon$ we can take two concentric spheres with radii $r_{1}$ and $r_{2}$, with $\left|r_{2}-r_{1}\right|$ very small, and by attaching $g+1$ disjoint Calabi handles we obtain a smooth orientable embedded surface of genus $g$, which is seen to be of geometrical degree 4.

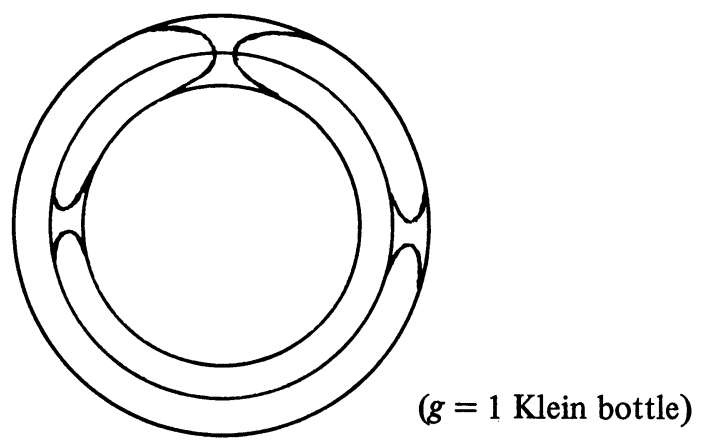

FIG. 3b

A smooth Klein bottle of geometrical degree six. By taking three concentric spheres with radii very close to each other, we can connect by suitably attaching $g+2$ Calabi handles and obtain a smooth immersion of geometrical degree six for any nonorientable surface of even Euler characteristic, $\chi(M)=$ $-2(g-1)$.

Real algebraic surfaces of algebraic degree 4. These have been studied and classified by Gudkov [2], Kharlamov [4], [5]. The sum of the Betti numbers $s \leqslant 20$, but $\leqslant 16$ for connected surfaces, so that the genus is $g \leqslant 7$. See Thom [9] and Milnor [8] for earlier but weaker inequalities. We can obtain these surfaces with formulas like (3.1). Here are examples.

$A$ "regular" surface with the symmetry of the cube, of genus 5 and degree 4 is given by

$$
\begin{aligned}
& \left(r^{2}-1\right)^{2}+\varepsilon^{4}(1+\varepsilon)\left(x^{4}+y^{4}+z^{4}\right)=\varepsilon^{4}, \\
& r^{2}=x^{2}+y^{2}+z^{2}, \quad \varepsilon>0 \text { small. }
\end{aligned}
$$

Observe that the unit sphere $r=1$ is interior to the surface $x^{4}+y^{4}+z^{4}=1$ and tangent at exactly 6 points where caps from the thick sphere are taken away. by

$A$ "regular" surface with the same symmetry of genus 7 and degree 4 is given

$$
\left(r^{2}-1\right)^{2}+\varepsilon^{4}(1+\varepsilon) \cdot \frac{81}{84} \sum_{e^{2}=f^{2}=1}(x+e y+f z)^{4}=\varepsilon^{4},
$$

where the analogous tangencies are at points $(x, y, z)=\frac{1}{\sqrt{3}}( \pm 1, \pm 1, \pm 1)$. 
Exercise. Find a regular algebraic surface of algebraic degree 4 of genus 3, with the symmetry of the regular simplex.

Algebraic surfaces of geometrical degree 4 of any genus g. $C^{4}$-robustness of geometrical degree. Those smooth surfaces of geometrical degree 4 and genus $g \geqslant 2$ which we constructed above can be assumed to have the following property.

A plane which is orthogonal to the tangent plane at a point $p$ of a surface is called an orthoplane at $p$, which meets the surface in an orthoplane section. In our examples the 4-jet of any orthoplane section at any point $p$ of the surface is not flat. This means that in suitable coordinates in the orthoplane the section has an equation

$$
v=\alpha_{2} u^{2}+\alpha_{3} u^{3}+\alpha_{4} u^{4}+\cdots, \quad \text { with }\left(\alpha_{2}, \alpha_{3}, \alpha_{4}\right) \neq(0,0,0) .
$$

This property is invariant under perturbations which are $C^{4}$-small: all derivatives of order 1,2,3, and 4 are small. This implies that locally the degree of our surfaces does not increase with $C^{4}$-small perburbations. Since in our examples, there is also no possible increase globally under such perturbations, the degree four remains unchanged. In other words, the degree four of our surfaces is a $C^{4}$-robust property. Then as we can $C^{4}$-approximate by (a component of) an algebraic variety, we find (locally-)algebraic models of geometrical degree four, for orientable surfaces of any genus $g$.

Exercise (Conjecture). If we choose $N$ sufficiently large, and then $\varepsilon>0$ sufficiently small, the following equation defines an algebraic surface of genus $g$ and (probably) of geometrical degree 4:

$$
\left(r^{2}-1\right)^{2}+\varepsilon^{4}(1+\varepsilon) \cdot \frac{1}{2} \sum_{j=0}^{g}\left(1+\cos \frac{2 \pi j}{g+1}+y \sin \frac{2 \pi j}{g+1}\right)^{N}=\varepsilon^{4} .
$$

\section{TIGHT smooth (even locally algebraic) orientable surfaces of geometrical degree six and any genus $g$}

We first construct smooth surfaces. Start from a round torus of degree 4 obtained by rotating a circle around a disjoint line in its plane. Any projective transform is also tight and of degree 4. Moreover the strictly convex part where $K>0$ can be modified at will as long as it remains strictly convex, i.e., $K>0$. Choose two points $p$ and $q$ on a round sphere. Place a projectively transformed standard torus such that its negative curvature-part approximates the line segment $p q$ and such that the two tangent planes along the points where $K=0$ cut the sphere, and are parallel to and near to the tangent planes to the sphere at $p$ and $q$. With a modification we can arrange that the convex part of the 
surface contains almost all of $S^{2}$ except two small neighborhoods $U_{p}$ and $U_{q}$ of $p$ and $q$. We have attached one handle to the two-sphere $S^{2}$ near $p$ and $q$ so that the geometrical degree of the surface obtained is four. The new surface is tight: at each point where the Gauss curvature is $K>0$, there is a globally supporting half-space $h$.

Next we attach $g$ such mutually disjoint thin handles near to chords $p_{j} q_{j}$, $j=1, \cdots, g$, to the round sphere, and such that no straight line meets more than two handles. We get a surface $M_{g}$ of genus $g$, which is tight and whose geometrical degree is seen to be six. The geometrical class is then $6-\chi\left(M_{g}\right)$ by Theorem 2 . See Fig. 4.

Robustness and algebraic examples. The degree six of these surfaces is as before a $C^{4}$-robust property. Tightness on the other hand is not robust at all. However, if we can keep some high order jet at the union of the plane (!) sets of points where $K=0$, fixed, then we have enough freedom for a $C^{4}$-small perturbation to obtain tight locally algebraic surfaces of geometrical degree six. Here is an explicit construction.

Let $f_{1}=0, \cdots, f_{g}=0$ be the polynomial equations of the disjoint projective transforms of a standard torus with $f_{j}>0$ representing the unbounded component of the complement for $j=1, \cdots, g$. Each torus has two highly supporting half-spaces given by linear inequalities in the coordinates

$$
h_{i} \geqslant 0, \quad i=2 j-1,2 j ; j=1, \cdots, g .
$$

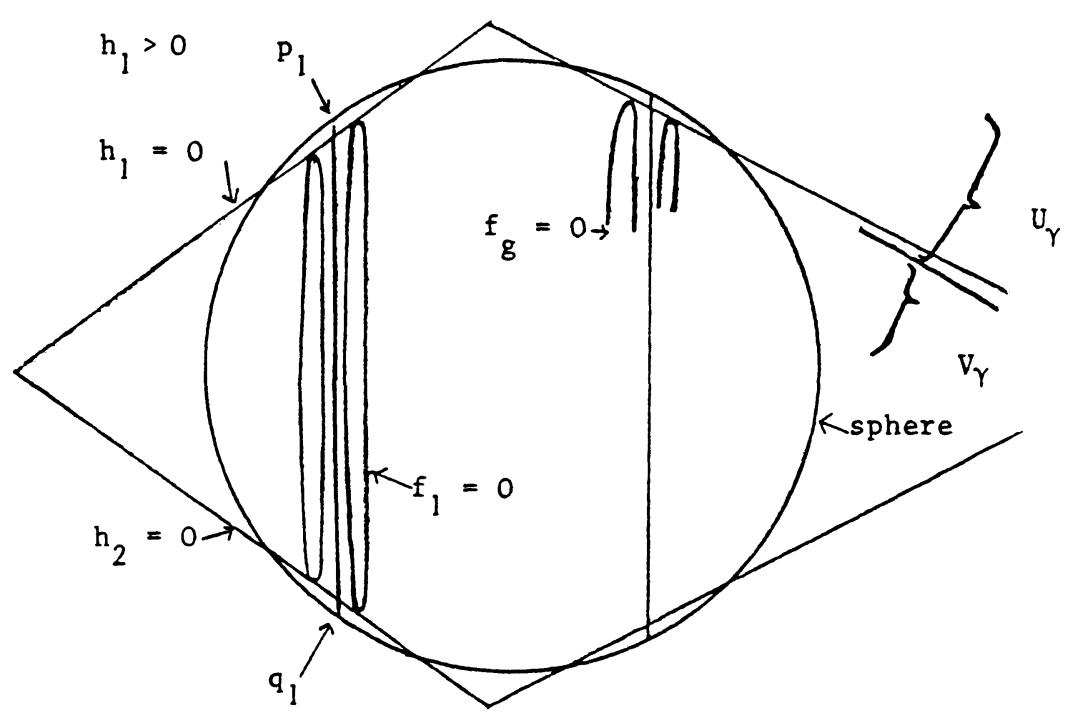

FIG. 4 
The smooth surface we constructed has an equation $f=0$ for some $C^{\infty}$-function $f$, for which we can assume by construction that it coincides with the polynomial function $\prod_{j=1}^{g} f_{j}$ in the union $U_{\gamma}$ of the two sets

$$
\left\{p: \sup _{j} h_{j}(p) \geqslant 0\right\}, \quad\left\{q: \prod_{i=1}^{2 g} h_{i}^{4}(p) \leqslant \gamma\right\}
$$

for some small $\gamma>0$. The complement $V_{\gamma}=E^{3} \backslash U_{\gamma}$ of $U_{\gamma}$ in $E^{3}$ is homeomorphic to an open ball.

Define the $C^{\infty}$-function $\varphi: E^{3} \rightarrow \mathbf{R}$ as zero on $U_{\gamma}$ and by

$$
\varphi=\frac{f-\prod_{j=1}^{g} f_{j}}{\prod_{i=1}^{2 g} h_{i}^{4}}
$$

on $V_{\gamma}$. Let $g$ be a polynomial approximation of $\varphi$ on a large compact convex set $W$, which contains $V_{\gamma}$ and the surface $M$, such that the sum of the squares of all derivatives up to and including those or order $k$ obeys

$$
\|g-\varphi\|_{k}^{2}<\varepsilon, \text { for some } \varepsilon>0,
$$

and at all points $p \in W$.

We had

$$
f=\prod_{j=1}^{g} f_{j}+\varphi \cdot \prod_{i=1}^{2 g} h_{i}^{4},
$$

and obtain the polynomial approximation on $W$

$$
f^{+}=\prod_{j=1}^{g} f_{j}+g \prod_{i=1}^{2 g} h_{i}^{4} .
$$

For $\varepsilon>0$ small and $k \geqslant 4$ we find the desired surface $M_{g}^{+}$of genus $g$ with the equation $f^{+}=0$, which $C^{4}$-approximates $M_{g} . M_{g}^{+}$is diffeomorphic with $M$, has the same points and 3 jets at each top-cycle-set $M \cap\left(h_{i} \geqslant 0\right)$, has Gauss curvature $K \neq 0$ outside these top cycle sets, is a component of an algebraic variety, is tight, and has geometrical degree six by $C^{4}$-robustness of degree under the given circumstances, as there are no global dangers for degree increases. The geometrical class $6-\chi\left(M_{g}\right)$ of $M_{g}^{+}$is unchanged.

Tight surfaces with large class and degree. Proof of Theorem 2a. Consider a point $p$ on a tight surface $M$ with $\chi(M) \neq 2$, at which the Gauss curvature is $K<0$. For any $C^{2}$-small perturbation near to $p$ the curvature remains negative, and the surface remains tight. There is however a perturbation, as $C^{2}$-small as we please, such that a tangent line $l$ in an asymptotic direction at $p$ contains some line segment of the perturbed surface $M^{\prime}$. With a second $C^{2}$-small perturbation of $M^{\prime}$ we can arrange the line $l$ to be transversal to the 
new surface $M^{\prime \prime}$ at as many points as we please. Then the degree and, by Theorem 1, also the class of our tight surface $M^{\prime \prime}$ are as large as we please. It could be made $\infty$.

\section{A TIGHT smooth embedding with geometrical degree four of a surface of genus two}

We construct a smooth surface $M=M_{2}$ of genus two with properties and symmetries as those of the example in Fig. 1b. It will turn out to be a component of the real algebraic variety of algebraic degree five with equation

$$
\begin{aligned}
& \{(x, y, z):-1 \leqslant z \leqslant 1\} \subset \mathbf{R}^{3}, \\
& 2 y\left(y^{2}-3 x^{2}\right)\left(1-z^{2}\right)+\left(x^{2}+y^{2}\right)^{2}=\left(9 z^{2}-1\right)\left(1-z^{2}\right) .
\end{aligned}
$$

Construction and geometry of $M$. Consider the function, on the $x, y$-plane,

$$
\eta(x, y)=2 y\left(y^{2}-3 x^{2}\right)+\left(x^{2}+y^{2}\right)^{2} .
$$

This function has the symmetry of the equilateral triangle, as $y\left(y^{2}-3 x^{2}\right)$ is the real part of $(y+i x)^{3}$. We want the level curves of our surface $M$ to be similar to the level curves of $\eta$. The graph of the function $\eta$ as a monkey saddle at $x=y=0$, and its asymptotic curves are nearly orthogonal to each other close to $(0,0)$. Some levels are given in Fig. 5a.

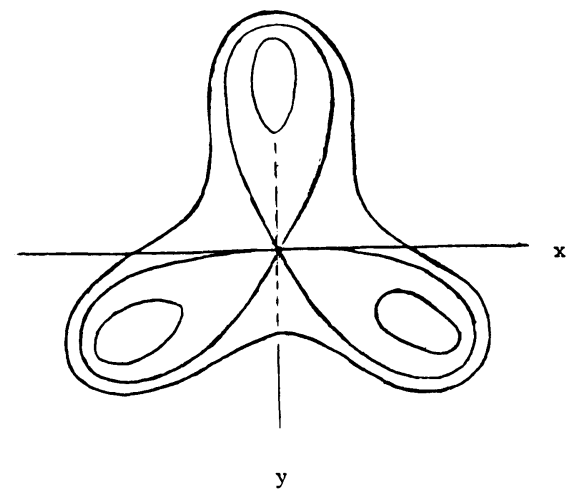

FIG. 5a

If we put $\eta=z^{2}-1$, we get monkey saddles at heights $z=+1$ and -1 , and three vertical tubes in between. But this surface does not close either above or below. We proceed to change levels by similarities. As in Fig. 1b we do want three highly supporting vertical planes for tightness. To prepare this we 
determine double tangents $y=c$ for each level curve of $\eta$, and we arrange by a similarity $x=c u, y=c v$ that these level curves are tangent to the vertical plane $y=1$. Then automatically by rotational-symmetry of order three, they are doubly tangent also to the other two corresponding vertical planes. Let us calculate the intersection of the plane $y=c$ and the level curve

$$
2 y\left(y^{2}-3 x^{2}\right)+\left(x^{2}+y^{2}\right)^{2}=\eta=\text { constant } .
$$

We get

$$
x^{4}+2 c^{2} x^{2}+c^{4}+2 c^{3}-6 c x^{2}-\eta=0
$$

that is,

$$
\left[x^{2}+c(c-3)\right]^{2}+c^{2}(8 c-9)-\eta=0 .
$$

In order for $y=c$ to be a double tangent we must have

$$
\eta=c^{2}(8 c-9) \text {. }
$$

For any $\eta$ we only want the largest value of $c$. Now we substitute $x=c u$ and $y=c v$ in

$$
2 y\left(y^{2}-3 x^{2}\right)+\left(x^{2}+y^{2}\right)^{2}=c^{2}(8 c-9),
$$

and find

$$
2 v\left(v^{2}-3 u^{2}\right) c^{3}+\left(u^{2}+v^{2}\right)^{2} c^{4}=c^{2}(8 c-9),
$$

or

$$
2 v\left(v^{2}-3 u^{2}\right)+c\left(u^{2}+v^{2}\right)^{2}=\left(8-\frac{9}{c}\right) .
$$

Finally we replace $(u, v)$ by $(x, y)$ and take

$$
8-\frac{9}{c}=9 z^{2}-1 \text {. }
$$

We get the equation announced in (5.1),

$$
2 y\left(y^{2}-3 x^{2}\right)\left(1-z^{2}\right)+\left(x^{2}+y^{2}\right)^{2}=\left(9 z^{2}-1\right)\left(1-z^{2}\right) .
$$

The monkey saddles are at heights $z= \pm \frac{1}{3}$. For $\frac{1}{9}<z^{2}<1$ the level curves at height $z$ is one closed curve. These curves converge for $z \rightarrow \pm 1$ to the points $(x, y, z)=(0,0, \pm 1)$. It then is geometrically seen that $(5.1)$ defines a closed surface $M$ of genus 2 .

The geometrical degree of $M$ is 4 . Any line which meets $M$ transversally must meet it in an even number of points, the ends of intervals in which the line meets the body $B$ bounded by $M$. This even number is at most four since the algebraic degree of (5.1) is five. So $M$ is of geometrical degree 4. 
Next we intersect the surface $M$ with the vertical plane $y=1$. We find the equation

$$
\left(x^{2}+3 z^{2}-2\right)^{2}=0
$$

of an ellipse $\varepsilon_{1}$ counted twice. It is the interaction of $M$ with the highly supporting vertical plane $y=1$. Analogous ellipses $\mathcal{E}_{2}$ and $\mathcal{E}_{3}$ occur in the vertical planes obtained from $y=1$ by rotation over $2 \pi / 3$ and $4 \pi / 3$ about the $z$-axis.

For making a nice model (Fig. 5b), we take $x, y$ and $3 z$ as unit orthogonal coordinates. The union of the three ellipses $\mathcal{E}_{1} \cup \mathcal{E}_{2} \cup \mathcal{E}_{3}$ divides the surface $M$ into two open parts $M^{+}$and $M^{-}$each of which is orthogonally projected into the horizontal plane $z=0$ with only folds along the intersection of $M$ with $z=0$. The points of $M^{-}$have $K<0$ except at the monkey saddle points where $K=0$. The points of $M^{+}$are on the boundary of the convex hull $\mathcal{H}(M)$, and have $K>0$ except at the top and bottom where $x=y=0$.

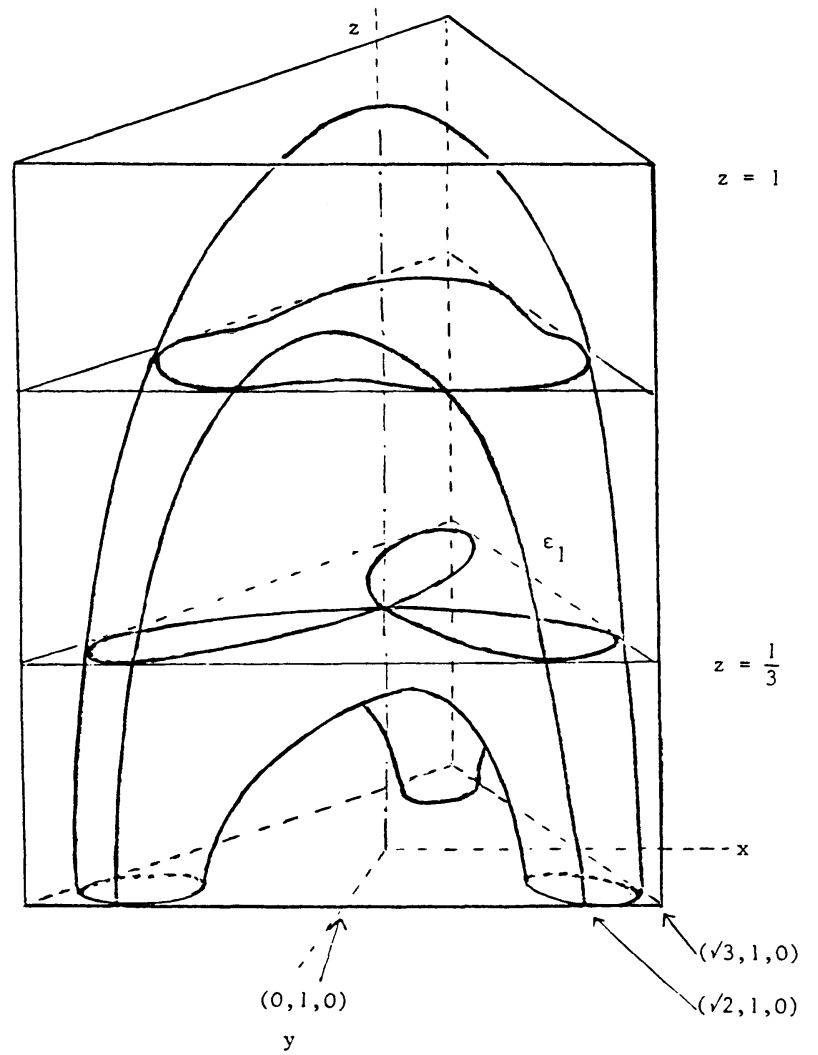

FIG. $5 b$ 
To check negative Gauss curvature $K<0$, we study convenient plane sections through any point $p \in M^{-}$, one for which the curvature vector points inside the body $B$, and one for which the curvature vector points outside $B$. This suffices for $K(p)$ to be negative. Here are sections which do this for us in large parts of $M^{-}$.

(i) Take vertical planes $y=c$. By looking at horizontal sections (!) we see that such sections are at most tangent at the obvious points in the plane of symmetry $z=0$ : In between, the isotopy type of the section does not change. For $c<1$, but close to 1 , the section consists of two closed embedded curves one inside the other. As the geometrical degree of the union is 4 , the inside curve is convex, and so its curvature vector points outside $B$. The same applies to the sections obtained from those discussed by rotation about the $z$-axis over $2 \pi / 3$ and $4 \pi / 3$. See Fig. 6 [(i) out], for the part of $M^{-}$covered by this case.

(ii) For horizontal plane sections we get three ovals for $9 z^{2}-1<0$, which can be shown to be convex. At the points covered (see Fig. 6 [(ii) in]) the curvature vector points inside $B$.

(iii) Also the vertical plane sections $y=\alpha x$ through the $z$-axis can be easily understood. They have horizontal tangents of the $z$-axis and that makes some of the parts of the section convex or concave leading to curvature vectors pointing inside. See Fig. 6 [(iii) in]. We do not elaborate the small remaining part of $M^{-}$.

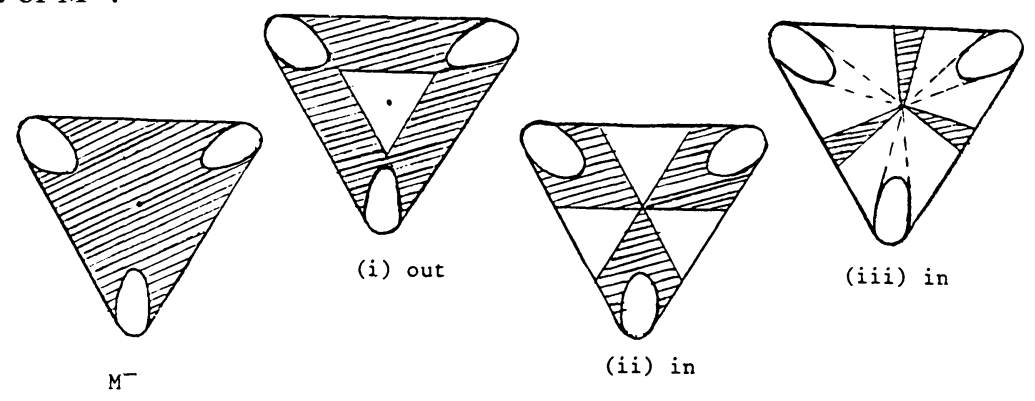

Fig. 6

All points of $\mathrm{M}^{+}$not on the $z$-axis have positive Gauss curvature $K>0$. Again by looking at vertical sections and horizontal sections one convinces oneself of this fact. We did not work out a formal calculation.

\section{References}

[1] T. Banchoff, Tight polyhedral Klein bottles, projective planes and Moebius bands, Math. Ann. 207 (1974) 233-1243.

[2] D. A. Gudkov, Russian Math. Surveys 29 (1974) 1-79.

[3] Y. Kergosien \& R. Thom, Sur les points paraboliques des surfaces immergées dans l'espace euclidien à trois dimensions, C. R. Acad. Sci. Paris 290 (1980) 705-709. 
[4] V. M. Kharlamov, The maximum number of components of a surface of degree 4 in $\mathbf{R} P^{3}$, Funkcional. Anal. i Prilǒzen 6 (1972) 101.

[5] _ Isotopic types of nonsingular surfaces of fourth degree in $\mathbf{R} P^{3}$, Funkcional. Anal. i Prilǒzen 12 (1978) 86-87.

[6] N. H. Kuiper, Morse relations for curvature and tightness, Lecture Notes in Math. Vol. 209, Springer, Berlin, 1971, 77-89.

[7] - Tight embeddings and maps. Submanifolds of geometrical class three in $E^{N}$, The Chern Symposium 1979, Springer, Berlin, 97-145.

[8] J. Milnor, On the Betti numbers of real varieties, Proc. Amer. Math. Soc. 15 (1964) 275-280.

[9] R. Thom, Sur l'homologie des variétés algébriques réelles, Differential and Combinatorial Topology (A Sympos. in Honor of Marston Morse), Princeton University Press, Princeton, 1965, 255-265.

[10] _ Sur les variétés d'ordre fini, Global Analysis (Papers in Honor of K. Kodaira), Princeton University Press, Princeton, 1969, 397-401.

[11] D. Hilbert \& S. Cohn-Vossen, Anschauliche Geometrie, Springer, Berlin, 1932, Fig. 288.

[12] E. Pitcher, Critical points of a map to a circle, Proc. Nat. Acad. Sci. U.S.A. 25 (1939) 428-431.

\section{BROWN UNIVERSITY}

InSTITUT Des Hautes Études SCIENTIFIQUes, France 